\title{
Incident-Driven Routing in Wireless Sensor Networks, a Cross-layer Approach
}

\author{
Mohammad R. Faghani and Uyen Trang Nguyen \\ Department of Computer Science and Engineering \\ York University, Toronto, Ontario, M3J 1P3 Canada \\ \{faghani@cse.yorku.ca\},\{utn@cse.yorku.ca\}
}

\begin{abstract}
Wireless sensor networks (WSNs) are being deployed widely thanks to recent advances in wireless communication technologies. Many WSNs may form in hostile environments, especially in military applications. Sensor nodes are thus prone to different types of attacks such as jamming, collision attacks, and eavesdropping. Once a sensor node is compromised, it is likely that the information passing through this node will be revealed to the attacker, or will never reach the destination (e.g., in jamming attacks). In this paper, we propose a cross-layer scheme that uses information from the application layer to locate compromised nodes, computes a new, secure path connecting the source and destination and routes data packets along the new path to the destination. We present our simulation and result visualization to show the effectiveness of the proposed incident-driven routing algorithm.
\end{abstract}

Index Terms - wireless sensor network, secure routing, crosslayer design.

\section{INTRODUCTION}

Wireless sensor networks (WSNs) are used ubiquitously in different types of applications from surveillance and monitoring to personal health care and navigation systems. In most of WSN applications, security is a major concern. Deployed in a hostile environment, especially in military applications, wireless sensors are prone to different types of attacks such as eavesdropping, jamming and collision attacks. Once a sensor node is compromised, it is likely that the information passing through this node will be revealed to the attacker, or will never reach the destination (e.g., in jamming and black hole attacks).

Although there exist solutions to the above problem, they are not practical to be applied to WSNs. For example, one solution to jamming attacks is channel surfing [10]. Nodes under attack will switch to another channel that is not being jammed. This requires some degree of coordination among nodes to select and tune in a common channel. This type of coordination consumes energy and requires high computational power, which is not suitable for sensor nodes. Another solution attempts to overcome the jamming condition by increasing power level or using more complex coding schemes in addition to prioritization of messages that a node sends [11]. This solution is also energy-consuming and computationally intensive.

In this paper, we propose a cross-layer scheme that uses information from the application layer to locate compromised nodes, computes a new, secure path connecting the source and destination, and route data packets along the new path to the destination. Sensors can take advantage of existing techniques $[19,20]$ to determine whether they are under attack. After that, they apply our proposed scheme for forwarding data to the destination via a secure route instead of the original path. We present our simulation and result visualization to show the effectiveness of the proposed incident-driven routing algorithm.

The remainder of this paper is organized as follows. We discuss related work in section II. In section III, we describe the proposed incident-driven routing algorithm. Simulation results are presented in Section IV. We summarize the paper and discuss our future work in Section V.

\section{RELATED WORKS}

Many secure routing protocols have been proposed for adhoc networks [7, 8, 10-14]. However, most of them cannot be applied directly to wireless sensor networks due to computational power and energy limitations of sensor nodes.

There exist also secure routing algorithms designed for adhoc networks that are based on public key cryptography [1014]. Nevertheless, public key cryptography is computationally intensive and not suitable for use in WSNs.

Routing algorithms based on symmetric key cryptography such as [15-18] are less intensive computationally. However, they are based on source routing or distance vector routing, and not appropriate for WSNs.

Greedy Perimeter State Routing (GPSR) [9] uses geographical locations of sensor nodes to establish routing paths. To route around an area through which the path cannot pass, the protocol tries to find the perimeter of the planar area. To do so, GPSR greedily sends out a packet for potentially many hops, before the packet loops and is recognized as undeliverable. The routing delay is thus potentially high in GPSR.

Our proposed algorithm, on the other hand, computes a secure path first and then sends packets along the new path, eliminating the delay caused by packet looping.

\section{The Proposed InCIDENT-DRIVEN Routing ALGORITHM}

In this section, we describe our proposed routing algorithm which, upon being notified of the location of an area under attack, will route data away from that area. In section III.A, we provide an overview of the proposed algorithm, including the computation of virtual coordinates and selection of nexthop candidate routers. We then present the next-hop node 
selection policy section III.B.

\section{A. Overview of the proposed routing algorithm}

We assume that the sensor nodes are capable of detecting anomalous activities around them. For example, wireless multimedia sensors have the video capability to detect and identify attackers [1]. Traditional sensor nodes, on the other hand, may use jamming and intrusion detection schemes [19, 20] to monitor their environment. Our proposed scheme is cross-layered in the sense that information from the application layer (video or jamming/intrusion detection confirmation) will trigger the execution of the proposed routing algorithm at the network layer.

Upon detecting an incident, a sensor sends an event notification message to its neighbors. This sensor is called a tainted sensor since there is a high probability that it is currently (or will soon be) under attack. A sensor counts the number of distinct event notification messages it received from its neighbors. The count allows the sensor to estimate how far it is from the incident. Generally speaking, the closer it is to the event, the more messages it receives.

We also assume that a receiver (sink) is associated with one or more backup receivers (sinks). When the main sink fails or is under attack, data will be routed to a backup sink. This type of deployment redundancy is a common practice in networking, for load balancing and combating node failures and security threats. Every node in the network is informed of the locations of all the sinks, and can compute its own location using a localization algorithm [9]. The node first computes the Euclidean distance to every sink, and then selects the closest node as its primary sink and the second closest node as the backup sink.

Our proposed routing algorithm uses three types of coordinates as in [4]: absolute coordinates, virtual coordinates and mapping coordinates. They are defined as follows.

Let $o$ denote the origin of the coordinate system (e.g., the south west corner of the physical deployment area), $t$ denote the sink, and $h$ denote the node on the routing path that wishes to select the next node to add to the routing path, as illustrated in Fig. 1. To initiate the next-node selection process, $h$ broadcasts its absolute coordinate $\left(x_{h}^{o}, y_{h}^{o}\right)$ to its neighbors. A neighbor node $i$ thus knows the position of its upstream node $h$, which is $\left(x_{h}^{o}, y_{h}^{o}\right)$, in addition to its own position $\left(x_{i}^{o}, y_{i}^{o}\right)$ and the sink position $\left(x_{t}^{o}, y_{t}^{o}\right)$.

The virtual coordinates of a node (e.g., node $i$ in Fig. 1) are defined as its coordinates in the virtual two-dimensional coordinate system where its upstream node (e.g., node $h$ in Fig. 1) is the origin, and the $X$-axis is the line connecting the upstream node $h$ and the sink. In the example shown in Fig. 1, the virtual coordinates of $i$ are denoted by $\left(x_{i}, y_{i}\right)$, and calculated as follows:

$$
\begin{aligned}
& \left\{\begin{array}{l}
x_{i}=\cos (\theta) \cdot\left(x_{i}^{o}-x_{h}^{o}\right)+\sin (\theta) \cdot\left(y_{i}^{o}-y_{h}^{o}\right) \\
y_{i}=\cos (\theta) \cdot\left(y_{i}^{o}-y_{h}^{o}\right)-\sin (\theta) \cdot\left(x_{i}^{o}-x_{h}^{o}\right)
\end{array}\right. \\
& \theta=\tan ^{-1}\left(\frac{y_{i}^{o}-y_{h}^{o}}{x_{i}^{o}-x_{h}^{o}}\right)
\end{aligned}
$$

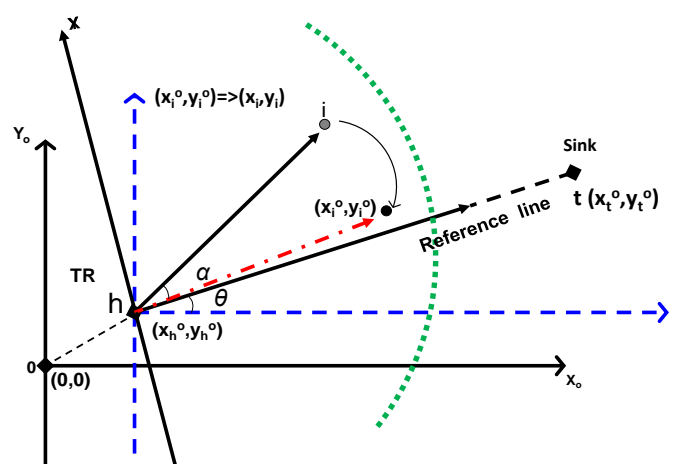

Fig. 1. Next hop selection in DGR [4]

We define ReferenceLine as a straight line connecting the origin of the virtual coordinate system (e.g., node $h$ in Fig. 1) to the sink; DeviationAngle $(\alpha)$ is the angle that specifies how much a path is expected to deviate from the ReferenceLine at the origin point. If we rotate the virtual coordinates around the origin by an angle $\alpha$, the rotated coordinates are called mapping coordinates. If $\alpha>0$, the rotation is clockwise; if $\alpha<0$, the rotation is counterclockwise. Moreover, $\alpha=0$ means that the path will be the shortest path along the direction from $h$ to the sink. The mapping coordinates are used during the path establishment process to determine a node's suitability of becoming the next node on the routing path. In the example shown in Fig. 1, the mapping coordinates of $i$ are denoted by $\left(x_{i}^{m}, y_{i}^{m}\right)$, and calculated as follows.

$\left\{x_{i}^{m}=\cos (\alpha) \cdot x_{i}+\sin (\alpha) \cdot y_{i}\right.$

$\left\{y_{i}^{m}=\cos (\alpha) \cdot y_{i}-\sin (\alpha) \cdot x_{i}\right.$

\section{B. Next hop selection policy}

To discover a direction-aware path, the source first broadcasts a probe message for route discovery. The selected next node will continue to broadcast the probe message to find its next forwarding node, and so forth. A probe message contains the following information (see also Fig. 2):

- $\quad$ source ID (SourceID)

- IDs of the primary sink (SinkID1) and backup sink (SinkID2)

- $\quad$ sequence number of this packet (SeqNum)

- deviation angle $\alpha$ as defined above (DeviationAngle). If $\alpha$ is a negative value, a path will be established below the ReferenceLine connecting the current node and the sink; otherwise, the path will be above the ReferenceLine.

- source-to-sink hop count $H_{s}$ (field SrcToSinkHopCount) which is the ideal hop count from the source to the sink. Let $R$ be the maximum transmission range of a sensor node and $D_{s r c}^{t}$ be the distance between the source and the sink. Therefore, $H_{s}=\left\lceil\frac{D_{s r c}^{t}}{R}\right\rceil$.

The above values in a probe message are set by the source and are not changed while the probe message is traversing the network. A probe message also contains the following fields whose values will be changed by intermediate nodes on the 
routing path:

- the hop count from the source to the current node (HopCount)

- the ID of the current node which is generating this probe message (PreviousHop)

- the absolute coordinates of the current node (AbsolutePosition)

Upon receiving a probe message, a node will calculate its virtual coordinates based on its upstream neighbor's position which is given in AbsolutePosition field of the probe message it just received. Then, mapping coordinates is calculated based on the virtual coordinates and the DeviationAngle using (2).

\begin{tabular}{|c|c|c|c|c|}
\hline \multicolumn{5}{|c|}{ Fixed Values } \\
\hline SourceID & SEC & & SinkID1 & SinkID2 \\
\hline \multicolumn{2}{|c|}{ Deviation Angle } & \multicolumn{3}{|c|}{ SourceToSinkHopCount } \\
\hline \multicolumn{5}{|c|}{ Variable Values } \\
\hline \multicolumn{2}{|c|}{ HopCount } & \multicolumn{2}{|c|}{ PreviousHop } & solute Position \\
\hline
\end{tabular}

Fig. 2. Information in a probe message

In Fig. 3, the point $(R, 0)$ is named the StrategicMappingLocation. This point is located on the ReferenceLine at distance $\mathrm{R}$ from a node $h$ currently looking for a next forwarding node. In practice, it is unlikely that the next hop neighbor of $h$ is located exactly at the StrategicMappingLocation. Hence, we select a neighbor of $h$ whose mapping coordinates are the closest to the StrategicMappingLocation [4].

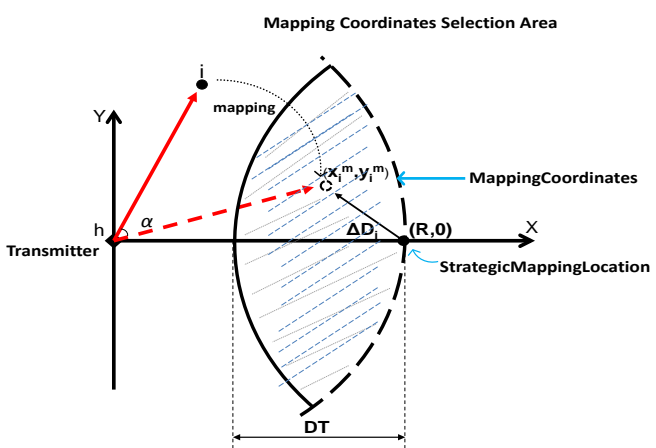

Fig. 3. Obtaining mapping coordinates [4]

The shaded area in Fig. 3 illustrates the neighbor selection area. The neighboring nodes whose mapping coordinates are located in this area are considered next hop candidates (NHCs).

Let $\Delta D_{i}$ be the distance between the StrategicMappingLocation and the mapping coordinates of node $i$ in the neighbor selection area. Thus, $\Delta D_{i}$ can be calculated as follows:

$\Delta D_{i}=\sqrt{\left(x_{i}^{m}-R\right)^{2}+\left(y_{i}^{m}\right)^{2}}$

To limit the selection area, a threshold, namely $D T$ is set. Node $i$ becomes an NHC if $\Delta D_{i}<D T$. In our proposed scheme, we exclude the NHCs that are located in the incident area. Conforming to this condition, the backoff time $t_{b}$ node $i$ has to spend before replying to the probe message from $h$ is calculated as follows [4]:

$t_{b}=\tau \times \Delta D_{i}+\operatorname{rand}(0, \mu)$,

where $\tau$ is a fixed interval, $\operatorname{rand}(0, \mu)$ gives a random value uniformly distributed in $(0, \mu)$ and $\mu$ is a small constant. Among the timers of the next hop candidates, the one with the smallest $t_{b}$ value will expire first and that node becomes the next forwarding node. Note that the smaller the $\Delta D_{i}$ value, the shorter the backoff time, allowing a node closer to the StrategicMappingLocation to be selected. If node $h$ has more than one neighbor with the same $\Delta D_{i}$ value, the random number $\operatorname{rand}(0, \mu)$ helps break the tie.

To enable the routing path to diverge from the area under attack, we proposed a new mechanism which adds an extra delay to $t_{b}$ to extend the response time, as follows:

$t_{b}=\tau \times \Delta D_{i}+\operatorname{rand}(0, \mu)+n \times \gamma$,

where $n$ is the number of received event notification messages and $\gamma$ is a constant value. The larger the value $n$, the closer the node is to the incident. Its timer is thus set longer so that it is less likely to be selected as the next forwarding node.

Let $i$ be the node with the shortest timer $t_{b}$. As soon as its timer expires, node $i$ will then send a unicast reply message (REP) to its upstream node $h$. To avoid collision among different REP messages at node $h, \tau$ is set to an adequately large value. Node $h$ will only accept the first REP message and ignores the subsequent replies. Upon receiving the first REP message, $h$ will broadcast a selection message (SEL) containing the ID of node $i$. All other NHCs hearing the SEL or REP message will stop their backoff timers. When node $i$ receives the SEL message containing its ID, it generates and broadcasts a probe message, and the above algorithm is repeated until the sink receives a probe message. The sink then broadcasts a confirmation message to terminate the path establishment process.

\section{Performance Analysis}

Routing away from the incident will obviously increase the length of the path. We now compute the increase in path length. In Fig. 4, the straight line $d$ represents the original path without applying our proposed scheme and the dashed arc $m$ is the traversed path after applying the proposed scheme.

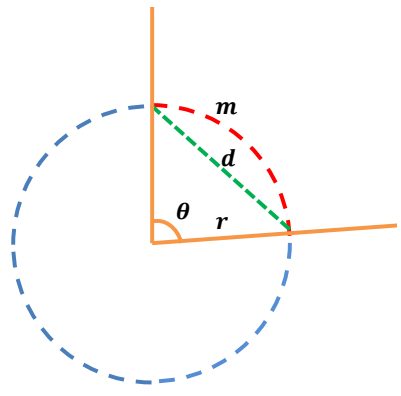

Fig. 4. Difference of the traversed path 
The values of $m$ and $d$ are calculated as follows:

$m=r \theta$,

$d=\sqrt{r^{2}+r^{2}-2 r^{2} \cos (\theta)}$,

where $r$ is the radius of the incident area and $0<\theta<\frac{\pi}{2}$ is a random. The difference in path length is thus:

$\Delta P_{1}=r \theta-\sqrt{r^{2}+r^{2}-2 r^{2} \cos (\theta)}$,

which is, on average, about $30 \%$ of the radius:

$\frac{1}{\pi} \times \int_{0}^{\pi}\left(r \theta-\sqrt{r^{2}+r^{2}-2 r^{2} \cos (\theta)}\right) d \theta=r\left(\frac{\pi^{2}}{2}-4\right) \frac{1}{\pi}$

Although the resulting path length is longer, our proposed algorithm allows data to reach the sink securely. In many cases, it is the only resort that allows data to reach the intended destination.

\section{Simulation RESUlts}

We used MATLAB for our simulations. The results are then visualized (Fig. 6 and 7) to show the effectiveness of the proposed algorithm.

In this experiment, 900 sensors are randomly distributed in an area of size $100 \mathrm{~m} \times 100 \mathrm{~m}$ as shown in Fig. 5 . The radio range of sensor nodes is $\mathrm{R}=10 \mathrm{~m}$. An incident took place at coordinates $(60,44)$, and all sensors within a distance of $25 \mathrm{~m}$ of the incident sensed the event. These tainted sensors are shown in the picture by red or dark colors.

A route request is received from the application layer to establish a connection from a source at coordinates $(0.6,43.7)$ to a destination at coordinates $(98.3,43.9)$, both in the purple color in the figures. If the route is established with an initial deviation angle of $\frac{\pi}{2}$, the connection will go through the area under attack as shown in Fig. 6.

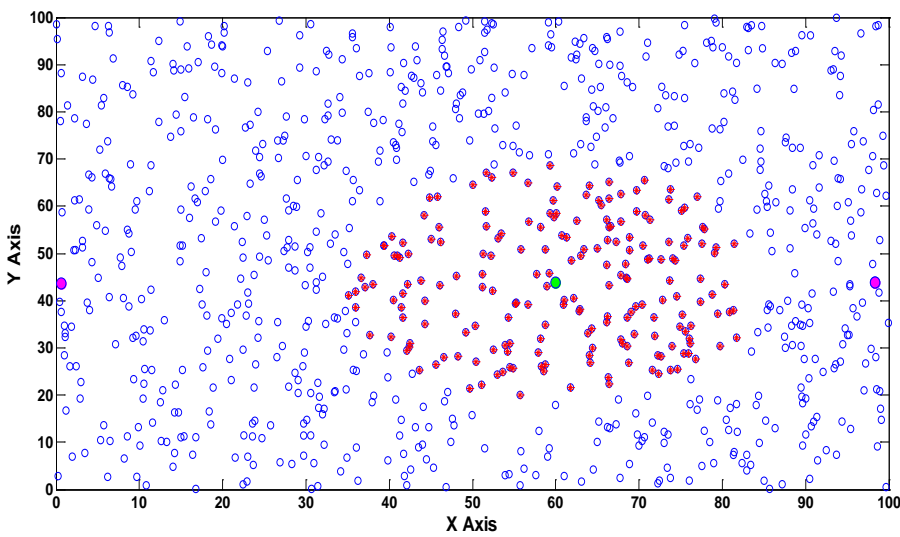

Fig. 5. Sensor field topology

Using the proposed scheme, each sensor in the area under attack will reply with a larger delay. This means that sensors that are not within that area are more likely to be selected. Fig. 7 shows the new routing path after the proposed routing algorithm is applied.

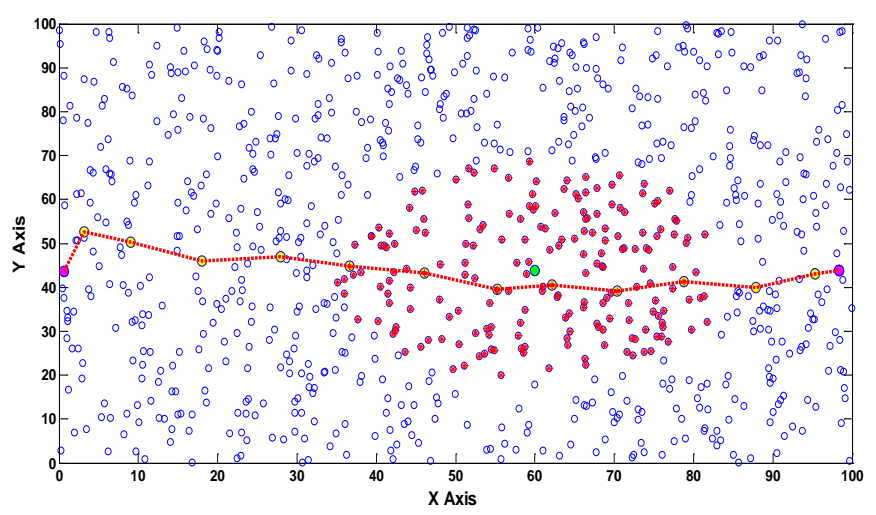

Fig. 6. Passing through incident area

As it can be seen, the new route bypasses the incident area to deliver data securely to the destination.

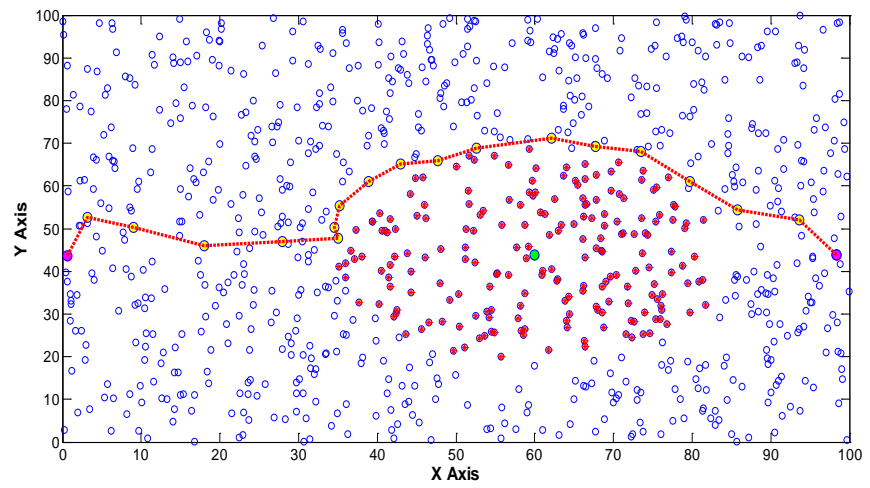

Fig. 7. Bypassing the incident area using the proposed scheme

\section{CONCLUSION}

Many WSNs are deployed in hostile environments such as battlefields. We propose a cross-layer algorithm that routes data away from the area under attack. The algorithm uses information from the application layer to locate compromised sensor nodes, and computes a new source-to-destination path that bypasses the compromised nodes and their surrounding area. Our simulation and visualization confirm the effectiveness of the proposed algorithm. Our performance analysis shows that the alternate paths are $30 \%$ longer than the original paths. However, that is the cost of routing data securely to the destinations when part of the network is under attack. In many cases, it is the only way to allow data to reach the destinations.

In our future work, we will conduct experiments to validate the analytical model presented above and to measure the additional overheads incurred by longer alternate paths. We will also consider the case in which the destination is located inside the area under attack.

\section{REFERENCES}

[1] M. Guerrero-Zapata, R. Zilan, J. M. Barcelo-Ordinas, K. Bicakci, and B. Tavli, "The Future of Security in Wireless Multimedia Sensor Networks: a position paper", Special Issue on Secure Multimedia Servicesll, Telecommunications System Journal (Springer), 2009 
[2] M. R. Faghani, and S. M. A Motahari, "Sectorized Location Dependent Key Management," Wireless and Mobile Computing, Networking and Communications, 2009. WIMOB 2009. IEEE International Conference on , vol., no., pp.388-393, 12-14 Oct. 2009

[3] Kundur, D.; Luh, W.; Okorafor, U.N.; Zourntos, T.; , "Security and Privacy for Distributed Multimedia Sensor Networks," Proceedings of the IEEE , vol.96, no.1, pp.112-130, Jan. 2008

[4] M. Chen, V. C. M. Leung, S. Mao, and Y. Yuan, "Directional geographical routing for real-time video communications in wireless sensor networks," Computer Communications, vol. 30, no. 17, pp. 33683383, 2007.

[5] K. Pelechrinis, M. Iliofotou, and S. V. Krishnamurthy, "Denial of Service Attacks in Wireless Networks: The Case of Jammers," Communications Surveys \& Tutorials, IEEE , vol.13, no.2, pp.245-257, Second Quarter, 2011

[6] D. Malan, M. Welsh, and M. Smith. A public-key infrastructure for key distribution in Tinyos based on elliptic curve cryptography. In 1st IEEE International Conference on Sensor and Ad Hoc Communications and Networks, pages 71-80, October 2004.

[7] K. Sanzgiri, B. Dahill, B. N. Levine, Clay Shields, E. M. Belding-Royer. A secure routing protocol for ad hoc networks. In proceeding of IEEE International Conference on Network Protocols (ICNP), 99(99), November 2002.

[8] M. Guerrero Zapata and N. Asokan. Securing ad hoc routing protocols. In Proc. ACM Workshop on Wireless Security (WiSe), pages 1-10. ACM Press, 2002.

[9] A. Srinivasan and J. Wu, "A Survey on Secure Localization in Wireless Sensor Networks," Encyclopedia of Wireless and Mobile Comm., CRC Press, Taylor and Francis Group, 2007.

[10] W. Xu, W. Trappe, Y. Zhang, "Channel surfing: defending wireless sensor networks from interference" In Proceedings of the 6th international conference on Information processing in sensor networks, pp. 499-508. ACM, New York, NY, USA (2007)

[11] W. Xu, K. Ma, W. Trappe, Y. Zhang, "Jamming sensor networks: attack and defense strategies”. Network, IEEE , vol.20, no.3, pp. 41- 47, MayJune 2006

[12] L. Zhou, Z. Haas, "Securing ad hoc networks", IEEE Network Magazine 13 (6) (1999) 24-30.

[13] F. Stajano, R.J. Anderson, "The resurrecting duckling: security issues for ad-hoc wireless networks", In proceeding of Seventh International Security Protocols Workshop, 1999, pp. 172-194.

[14] J. Hubaux, L. Buttyan, S. Capkun, "The quest for security in mobile ad hoc networks", In Proceedings of the ACM Symposium on Mobile Ad Hoc Networking and Computing (MobiHOC 2001), 2001.

[15] Y.-C. Hu, D.B. Johnson, A. Perrig, "SEAD: Secure efficient distance vector routing for mobile wireless ad hoc networks", In Proceedings of the 4th IEEE Workshop on Mobile Computing Systems and Applications (WMCSA 2002), 2002, pp. 3-13.

[16] Y.-C. Hu, A. Perrig, D.B. Johnson, "Ariadne: secure on demand routing protocol for ad hoc networks", in proceeding of MOBICOM, 2002.

[17] S. Basagni, K. Herrin, E. Rosti, D. Bruschi, "Secure pebblenets", In proceeding of ACM International Symposium on Mobile Ad Hoc Networking and Computing (MobiHoc 2001), 2001, pp. 156-163.

[18] P. Papadimitratos, Z. Haas, "Secure routing for mobile ad hoc networks", In proceeding of SCS Communication Networks and Distributed Systems Modeling and Simulation Conference (CNDS 2002), 2002.

[19] W. Xu, W. Trappe, Y. Zhang, T. Wood, "The Feasibility of Launching and Detecting Jamming Attacks in Wireless Networks," In Proceedings of the ACM International Symposium on Mobile Ad Hoc Networking and Computing (Mobihoc), 2005

[20] G. Thamilarasu, A. Balasubramanian, S. Mishra, R. Sridhar, "A crosslayer based intrusion detection approach for wireless ad hoc networks", In proceeding of Mobile Adhoc and Sensor Systems Conference, 2005. IEEE International Conference on , vol., no., pp.7 pp.-861, 7-7 Nov. 2005 